This is an accepted manuscript of an article published by SAGE Journals in Convergence: The International Journal of Research into New Media Technologies on October 18, 2016, available online: https://doi.org/10.1177/1354856516673298

\title{
Social streaming? Navigating music as personal and social
}

Anja Nylund Hagen and Marika Lüders (Department of Media and Communication, University of Oslo, Norway)

\begin{abstract}
Music-streaming services embed social features that enable users to connect to one another and use music as social objects. This paper examines how these features are experienced within negotiations of music as personal and social through the acts of sharing music and of following others. The analysis relies on 23 focus-group interviews with 124 Spotify or/and Tidal users, and a mixed-method study including music-diary self-reports, online observation, and interviews with 12 heavy users. Our findings suggest that users incorporate social awareness in non-sharing, selective-sharing, and all-sharing approaches with strong, weak, and absent ties. These ties are characterized by different configurations of social and music homophily. Negotiations of music as personal and social shape how music-streaming services are experienced.
\end{abstract}

\section{Keywords}

Music streaming, Spotify, Tidal, self-performance, taste cultures, networked individualism, weak ties, strong ties 


\section{Introduction}

Music-streaming services such as Spotify and Last.fm embed social features to enable users to connect with one another and use music tracks as social objects. Whereas music has always been social (Van Dijck, 2007), little is known about how people use social sharing features as part of music streaming. In Norway, where this study was conducted, Spotify and Tidal (previously WiMP Music) are particularly popular, and in 2015 streaming revenues accounted for 77.4 percent of all recorded music revenues in Norway (Ingham, 2016).

Music sharing in streaming services is multifaceted. In this article we highlight music sharing in terms of how users are able to connect with one another within the streaming service. Spotify and Tidal users can follow selected Facebook friends and receive feeds of music from them. Follow/following is currently asymmetrical, so that followed friends do not have to follow back. Spotify and Tidal users can also share music tracks and playlists as posts on Facebook and Twitter, or in email or SMS. Both services enable users to play music in 'private mode'. Social connections are hence embedded in these services in a two-way fashion. Sharing music is bound up with either explicitly or implicitly giving music recommendations; conversely, users can follow or browse others' pages and find music recommendations. In this paper we will look at how streaming services are experienced as social from the related perspectives of sharing and of following others, arguing that social sharing features align with the personal need to navigate and explore the vast catalogue of available music, yet challenge the experienced need to control self-performances.

Music listening is a communal and personal experience (Jones, 2011), and sharing music demands an internal reckoning regarding what is appropriate to share and what ought to be kept private. Any study of online music sharing hence necessitates an examination of how music connects with identity. In what follows, we will review relevant research and link previous studies with relevant theoretical perspectives on 
identity, self-performance, and social ties, in order to construct our analytical framework and derive our research questions.

\section{Sharing music: from self to peers}

The act of sharing music with friends and peers in music-streaming services instigates theoretical reflections of music as personal, and how streaming-users consider their own experiences of social interaction in music-streaming services, from the perspective of self-performances as reflexive and social practices.

Goffman's ([1959] 1990) theatrical metaphor of self-performances suggests that the self is shaped and staged according to contexts; we consciously edit the impressions we give, and attempt to control expressions given off. Our sense of self emerges through social interactions with others, and is a product of our own internal definitions, generated via a dialectical interplay with how we perceive that others define us (Blumer, [1969] 1998; Mead and Morris, [1934] 1967). In modern society, self-identity is likewise viewed as an inescapably reflexive project dedicated to assessing questions about who we are, where we come from, and whom we relate to (Giddens, 1991). These existential questions relate to Giddens' notion of ontological security, a psychological state based on individual's trust in others and a low or manageable level of anxiety. In everyday social life, ontological security derives from our degree of control over predictable routines and encounters (Giddens 1984: 64).

In networked publics, different social contexts often collapse, which complicates self-performance (Marwick and boyd, 2011). This context collapse potentially constrains acts of sharing music. The music we listen to often links to our sense of 'inner self', expressing our current state of mind (Liu and Reimer, 2008), influencing our mood, and giving meaning to our everyday life and routines by organizing experiences and acting as a symbolic referent for actions, experiences and feelings (DeNora, 2000; Turino, 1999; van Dijck, 2007). Music suggests interpretations and evokes memories so 
powerfully that the full details of one's listening practices may be too intimate to be shared (Jones, 2011). Voida et al. (2005: 194) find that listeners sharing music with others on their subnets in iTunes negotiate 'what identity to portray through one's own music library,' then note that this type of identity work recalls Goffman's perspective on impression management (Goffman, [1959] 1990). Relatedly, theories on reflexive selfperformances are prevalent in studies of personal music consumption, pointing to how music collections reveal autobiographical traces and are valued as markers of identity (Giles et al., 2007; Kibby, 2009).

Whereas music as personal may constrain sharing-practices, music listening also promotes a sense of belonging and relates one's sense of self to one's larger community and even one's 'generation' of peers (Van Dijck, 2007). As markers of identity music links to how we relate to contemporary heterogeneous taste cultures; with cultural taste being a means for ritual identification and of constructing social relations (DiMaggio, 1987). With music-streaming services music can technically easily be shared as taste statements for an extended network of peers. Aligning with Tepper and Hargittai's (2009: 230) observation regarding online music discovery, music-streaming services certainly facilitate an eclectic musical palette that likely relates to cultural capital, opinion leadership and social distinctions also when it comes to the users' ability to share music in streaming services. The links between identity, taste and status are likely nuanced and go beyond simple high-brow/low-brow distinctions (DiMaggio, 1987; Lizardo, 2006), and 'there are distinct social advantages to having broad tastes or broad knowledge about diverse cultural offering' (Tepper and Hargittai, 2009: 230). Broad taste cultures, however, require 'culture-switching' with people deploying taste selectively in different interactions and contexts (DiMaggio, 1987), echoing Goffman's theatrical metaphor of socially dependent self-performances.

To recap, we may hence expect to see tensions in individual patterns of sharing music. Music as personal may constrain acts of sharing, yet music as taste statements 
may motivate acts of sharing. Subscribing to the fact that people are reflexively aware of their self-performances (Giddens, 1991; Goffman, [1959] 1990), we will address how shareable music played through streaming services is.

\section{Exploring music: from peers to self}

Music listening and discovery practices are distinctively social, and people often rely on friends to discover new music (Laplante, 2011; Mesnage et al., 2011). In the pre-digital era, the sharing of music preferences happened face-to-face, but it also connected to technology via, for example, the preparation and sharing of mix tapes (Bitner, 2009; Jones, 2002). In the digital era, close friends remain important for music exploration (Komulainen et al., 2010; Laplante, 2011; Tepper and Hargittai, 2009). Yet, music has also become meaningful shareable objects that in turn instigate social interactions among friends and acquaintances in social network sites (SNSs) (Komulainen et al., 2010; Leong and Wright, 2013). As music-streaming services are often integrated with Facebook, where users maintain strong as well as weak ties (Brandtzæg, 2012; Ellison et al., 2007), it becomes consequential to study whom people turn to when seeking new music. The social significance of music implies there is a need to understand key notions and characteristics of social relations, and how networked technologies and streaming services, potentially expand whom we consider to be our musical peers.

Social dynamics and group-formation in modern societies are often explained and studied as consequences of the principle of 'likes attract' (Centola et al., 2007). Homophily, the tendency to prefer friendships or other kinds of bonds with similar rather than dissimilar others, has been found to be a consistent and prominent explanation for the formation of relationships (for a review, see McPherson et al., 2001). Whereas globalization and the advent of networked technologies expand access to people and ideas worldwide, structural patterns of interaction are not dissolving into a homogenous global culture; online as well as offline there is 'a strong tendency of 
people to self-organize into culturally defined groups' (Centola et al., 2007: 925). Notice the need for considering homophily and taste cultures in tandem, with the latter emphasizing the importance of popular cultural forms for connecting individuals to looser social networks (Lizardo, 2006).

In music-streaming services, homophily may privilege similarity or resonance of music interests. Baym and Ledbetter (2009) find that Last.fm friends share musical tastes even when their social ties are otherwise weak. Yet we have little knowledge of the significance of weak vs. strong ties in discovering music in streaming services. Granovetter (1973: 1361) distinguishes between strong, weak, and absent ties and defines the strength of a tie as resulting from a 'combination of the amount of time, the emotional intensity, the intimacy (mutual confiding), and the reciprocal services which characterize the tie'. In retrieval and exchange of information the strength of weak and absent ties derives from the direct links they provide to non-redundant information.

Granovetter's theory of ties is complemented by the concept of networked individualism, which accounts for the ways in which networked technologies liberate us from settled groups and allow us to navigate among multiple networks (Rainie and Wellman, 2012). Whereas geographical proximity remains important for the formation of ties, networked technologies allow greater geographical latitude in the formation of homophilous ties (McPherson et al., 2001). Networked technologies have allegedly enabled the emergence of limited-purpose and more fluid social networks (Benkler, 2006), and the possibility of navigating networks changes the way one accesses information. Social networks benefit the individual, and those with diverse networks, ones that include many weak-ties, are able to access information, support, and advice from more, and more diversified, sources (Rainie and Wellman, 2012). We may hence expect that music listeners in music-streaming services turn to an extended network of both strong, weak and absent ties as social and/or musical peers when exploring and seeking new music. 


\section{Research questions and analytical framework}

Given the personal nature of music and its frequent investment with memories and emotions, any inquiry of music sharing practices must consider the boundaries between personal and social. We therefore ask: Why do users choose to share or not share music, and how do they negotiate the need to balance music as personal and social?

Existing literature points to the importance of strong ties for discovering music, yet through SNS-integrated streaming services, users relate to close friends as well as peripheral acquaintances. With streaming services, we might expect to see an embrace of weak and absent ties as important recommenders. In this regard, we ask: Why do users follow strong, weak, or absent ties in streaming services?

These questions demarcate an analytical framework whereby personal and social streaming practices can be accommodated within the same register, and allow a discussion of whether music listeners regard music streaming as social. Based on the above literature review, we can summarize the expected patterns as follows:

1. Music as a personal experience makes it a valued social object to share, yet it also ties to notions of identity and social distinctions. This challenges individual music sharing patterns in general, and in music-streaming services in particular, because users connect with strong as well as weak ties in the social streaming network.

2. Streaming services afford new opportunities to discover new music through weak and absent ties in ways that were much less prevalent in the pre-digital age.

\section{Method}

This explanatory case study (Yin, 2009) relies upon two sets of qualitative data. For the first set, we conducted 23 focus-group interviews with 124 users of Spotify and/or Tidal, aged 18 to 59; between 2010 and 2013 (66 participants were male, 58 were female, average age 29 , median age 27). These informants were recruited at the 
pop/rock festival Øya in Oslo, Norway. The interviews were scheduled after the festival and conducted face-to-face. In the focus group interviews we asked the participants about their use of music-streaming services; what music means to them; how they explore and discover music; and how they relate to and use the opportunities to share music with others for example in the form of public playlists and sharing to SNSs. Focus group interviews were recorded, transcribed verbatim, and then coded and analysed using HyperResearch.

The second set of data involves findings from a self-reported study with 12 dedicated users of music-streaming services, ages 17 to 60 , writing diary entries about their own music streaming during four periods of two days each in March and April 2013. Participants who had used streaming daily for at least one year were recruited after visits to three high schools in the Oslo area, Norway, and after an announcement of the study on Facebook and Twitter. The self-reported study aimed to capture streaming experiences when and where they occurred, according to the dictates of the Experience Sampling Method, which seeks to avoid the potential distortions of retrospective inquiries (Hektner et al., 2007).

The diary entries were followed up by individual face-to-face qualitative interviews that lasted between 40 and 60 minutes. These interviews were recorded, transcribed verbatim, and coded and analysed using HyperResearch. The Facebook profiles and Last.fm scrobble logs of the diary participants were also monitored. The diary study contributed more contextual findings than the focus-group interviews and enabled us to consider streaming practices as well as streaming-related claims and statements from participants.

Our recruitment of informants at a music festival for the focus-groups and heavy users of music-streaming services for the diary-study means that they were all likely to cultivate an above-average interest in music; they were also relatively young and urban. Our study is hence limited in terms of studying a relatively homogenous group of 
streaming users in a country currently boasting one of the world's leading musicstreaming markets. Still, we believe the data provide insights into the habits of early adopters and avid users of music-streaming services.

We applied a simple deductive pattern-matching logic to our empirical material and compared the patterns we found in the data to the expected patterns described in the analytical framework section (Yin, 2009). We conducted a thematic analysis to generate codes and categories from the data. A thematic analysis allows for general issues to be determined prior to the analysis, yet the specific nature of the codes and categories arises in the process of coding the data (Ezzy, 2002). Beginning with the focus groups conducted in 2010, we assigned initial codes to the data through open coding. We discussed and amended these codes, then coded the interviews conducted in 2011 to 2013. For these focus groups, we developed additional codes to accommodate small changes in the interview protocols and themes discussed. The interviews with the diary participants were coded in a similar manner. As a final stage, we analysed how the themes that emerged through our coding and analysis fit the patterns we derived from relevant literature and theory (Ezzy, 2002).

\section{Findings}

Sharing music

Table 1 summarizes how the participants chose to share or not share music, and how sharing practices often relate to music-listening as personal. 
Table 1: Summary of sharing-types from focus groups and diary study.

Sharing-type $\quad$ Number of participants and typical motivations/reasoning

Share-all: 21 participants: 8 female, 13 male, average age: 29

Share everything with Music missionaries: want everyone to know about great music.

everyone. $\quad$ Efforts in curating playlists make them relevant to share.

Never use private sessions. Sharing music as a catalyst for future conversations.

Share to social media. No filters: do not care about other people's opinions.

Open as default setting, do not see the need to restrict.

Followers and feedback motivate sharing.

Share selectively: 80 participants: 42 female, 38 male, average age: 28

Selective playlists shared Motivations for sharing:

with everyone. Sharing as a gift, not for attracting followers. Sharing directly

Direct sharing with with friends with similar taste in music as an act of friendship.

selective friends. Sharing as catalyst for future conversations.

Occasionally use private Collaborative playlists with certain friends.

sessions. Music missionaries: want everyone to know about selected

Share selected music in music.

social media. Efforts in curating playlists make them relevant to make public.

Active and conscious self-presentation.

Motivations for not sharing: 
Impression management and insecurity about how others will judge their music. Guilty pleasures are kept private.

Some music regarded as too personal and revealing to share.

Context-collapse makes sharing difficult.

Some playlists regarded as irrelevant for others, e.g., lists with narrow track coherence or specific-purpose lists (such as workout music).

Not sharing to keep music exclusive to oneself.

Non-sharers: 30 participants: 13 female, 17 male, average age: 29

Do not share recently

played music either in

Spotify/Tidal or on

Facebook/Twitter.

Have no public playlists.
Sharing music is too personal.

Self-performance, social reputation, insecurity about how others will judge their music.

The account is not arranged systematically; lack of control over the content in playlists.

Not interested in sharing. Sharing is too much hassle. Use music streaming services for listening to music and nothing else.

Sharing is spamming and showing off.

Prefer to recommend and talk about music face-to-face.

Uncategorized: 5 participants could not be categorized based on the interviews. 
In the following analysis, we will discuss the ways in which music listening as personal both motivates and restricts sharing. We will further address how music sharing, social connections and impression management are linked.

Sharing and not sharing because it is personal. Annie (21) links music to life events in a way that resonates with research on the personal significance of recorded music in the pre-streaming era. Streaming services do not imply any change in her emotional investment to music or in the ways in which music moves her. She connects discovering and listening to music to particular experiences in her life to such an extent that sharing music can be meaningless:

Annie (21): If you [discover] music in another country or another place, and you develop a close relationship to it, and you share it with someone, and they haven't been there or experienced any of it, [then] they don't really understand the song or the artist. (...) There are things that are personal with music you have discovered yourself.

This is not to say that all of the music Annie listens to is intertwined with particular life events, and she shares certain selected playlists. Most of our informants likewise connected at least some music experiences with life narratives and memories. For nonsharers, music listening was deemed too personal and intimate an activity to be shared at all. Billy (39) says: 'When I listen to music, nobody knows what I'm doing. And I like it to be that way.' Non-sharers see music sharing as a way to show off. Their primary motivation is to listen to music, not to invite others into their personal musical universe.

Participants in all three groups listed in Table 1 (all-sharers, selective sharers and non-sharers) made efforts to save or stabilize their music listening by creating playlists to combat the tendency for their listening to become fragmented and ephemeral 
through music-streaming services. Playlists can mirror personal histories and can be curated to reflect everyday life tasks and moods. Sorting music into playlists consolidates one's personal ownership of music, even in a streaming context. For selective sharers and non-sharers, unshareable tracks and playlists include music that is too personal, intimate, or at odds with one's desired self-presentation, as well as music that seems irrelevant to others. A common argument against sharing is to protect one's contacts from too much unnecessary information, and sometimes shared music feeds are thought of as spam. Whether or not playlists are made public thus depends on their perceived shareability. Share-all participants regard all of their playlists as shareable, whereas selective sharers prepare only certain playlists for sharing, as Felix (23) describes: 'Sharing music is very important. I try to make public playlists that can easily be found and with names that convey what kind of music they include.'

Whereas music can be too personal or profoundly significant to be shared, these same qualities also sometimes argue precisely for its shareability. Music constitutes a valued social object, for specific friends, at least. Selective sharers emphasize the importance of sharing music as a way to strengthen social bonds and spark future interactions:

Eva (27): Music that I like a lot, I want to give it to people I like a lot (...). 'I like listening to this, it's so nice'. It creates a bond.

Andreas (25): It's kind of a catalyst for a future conversation. You send a link, and then you meet at a party later, and it's like, 'Oh, you saw that', and you're on.

In this case, sharing becomes more meaningful if it is 'effective', as is indicated by Andreas's comment, 'Oh, you saw that.' Music as a shared social object is like a gift, as diary participant Nina (27) writes: 'You know what, he added the track [I had recommended] to his playlist with [his] personal favourites! It's like, YES!' 
Friendships are characterized by a history of mutual trust and shared experiences that supply a feeling of belonging. People tend to select friends who are like they are (McKenna et al., 2002; McPherson et al., 2001), and in this context music is a social object that contributes to shared experiences and strengthens social bonds. Spotify and Tidal provide opportunities to share selectively with chosen friends, yet as we will see next, not everyone is comfortable with more public forms of sharing.

Sharing and impression management. The connection between music and impression management, as emphasized by Voida et al. (2005), is confirmed by our findings. Diary participant Erik (18) worries that his network would derive an inaccurate impression of him if people saw his devotion to pop ballads that 'someone else finds strange and depressing. I do not know if this is considered as typical boy music [either].' The need to cultivate a certain social reputation and avoid being judged on the basis of one's musical taste evokes the human need to maintain ontological security (Giddens, 1984). Non-sharers achieve this state simply by not sharing music, a decision often motivated by a desire for controlled self-exposure in one's interactions with peers. Sharing with confidence and trust requires more effort in terms of arranging and sorting playlists and toggling private streaming modes.

Selective sharers capitalize upon the social and symbolic roles of music in ways that steer sharing patterns and impression management. Motivations for making music public therefore include elements of reflexive self-presentation, with personal musicexposure regulated according to expectations of how others will perceive the music, or them, as a consequence of sharing. These users share particular recommendations and playlists, curated with effort and ingenuity, when they want to be associated with this music, or when music preferences are considered appropriate taste statements; shareable for an extended network of peers. 
Tom (36): It's like, in Facebook, you share what you consider cool, right? Like, if you find something obscure, you share it.

David (24): If I'm cleaning the house, I typically listen to a lot of crap, or what I would normally characterize as crap. And then I turn off the sharing function.

The latter quote exemplifies how switching to a private session is part of selective sharing. Like David's listening mode when he is cleaning, other listeners pointed to streaming moments that they were less inclined to share. For example listening with the purpose of experimenting or exploring new music can be risky in terms of impressions management.

John (35): You have so many playlists that are basically nonsense anyway. (...) You need to maintain a certain integrity, right? Last.fm was mentioned, and I think what's kind of defining 'guilty pleasures' is when you go and delete Last.fm updates.

With the integration of Spotify and Tidal with Facebook, listening patterns are potentially visible to different segments of one's friends. The consequential context collapse (Marwick and boyd, 2011) raises tensions in terms of 'culture switching' (DiMaggio, 1987). The social benefits of a broad repertoire of taste relate to cultural taste as interactional currencies for different types of relationships (DiMaggio, 1987; Lizardo, 2006). In the flattened social context of music-streaming services, there are limited opportunities to deploy taste according to context. Yet, share-all informants show enough confidence in their own musical tastes to share everything. Their confidence seems unaffected by the limited opportunities to tailor music taste statements to social context. 
Diary participant Kristoffer (21) shares all of his music and has become an important absent tie for thousands of followers. Specific genre searches in Spotify turn up several of Kristoffer's playlists as top suggestions, and they continually accumulate followers. His most popular list had nearly twenty thousand followers in May 2013. Most of these people are, of course, absent ties, reflecting a network formed around shared interests. Kristoffer rarely responds to messages from these followers or follows any of them in return, and they have no relationship history together besides a playlist connection. Yet Kristoffer is aware of his role as music provider for thousands of absent ties who are presumably also aware of him, in terms of receiving notifications about his playlist activity. Sometimes years old and far too numerous to engage with personally, these tie relations are of a permanent yet absent nature, resembling the asymmetrical and para-social relations that are typical of mass communication (Horton and Wohl, [1956] 1986).

Other share-all participants come across as cultural omnivores (Lizardo, 2006; Tepper and Hargittai, 2009), who are either confident or seemingly indifferent to how music as shared taste statements connect with taste cultures. They typically revel in the fact that they have no filters, literally or figuratively, and appear to experience no need for 'culture switching':

Anne (29): I have my complete Spotify profile [set to] public. Of course, like with regard to [my music] credibility, there's an awful lot there that shouldn't be public. But I don't think about it.

Mathias (28): Instinctively, I was like, 'No, no, no, nobody must [see]', but I share too much about myself anyway ... I don't care, I like to see what other people are listening to, and I have no shame with regard to what I'm listening to [laughs]. 
Share-all participants explain that they take an ironic approach to their own music listening or claim that they are not affected by judgments regarding their music listening, even when their sharing reveals guilty music pleasures. These listeners appreciate and value music, but do not see it as revelatory of anything outside itself. Often they are simply confident enough to endorse whatever impressions their playlists might make. Guilty pleasures can even be regarded from a positive flip-side demonstrating self-confidence confirmed with rewards in followers and likes, and hence absolutely right with regard to impression management. As Daniel (26) says, 'I've noticed [that for] more and more people, like, it becomes cool to listen to things that are uncool.'

To recapitulate our findings with regard to why users choose to share or not share their music and how they negotiate music as both personal and social, for most of our informants, certain music is unshareable because it is too personal and certain music is unshareable because it does not convey appropriate taste statements aligned with a preferred impression management. Music that is at odds with constructive impression management is less shareable, except for the committed share-all participants. Music's perceived intimacy and transparency in terms of life events act to inhibit sharing, but also make music into a valued social object for sharing with one's close ties, as an act of friendship, a reflexive selective self-performance, or the gesture of a music missionary. Following weak, strong, and absent ties in the context of exploring music Following, in general, means linking to social-network ties via service features that produce and display updates and feeds of peers' current activity. We use the related notion of indirect following to refer to paying attention to or tracking ties without formally following them. Both direct and indirect following draw upon insights gained from accessing peers' listening patterns to discover music. Following in streaming services is asymmetrical, which means that participants experience sharing and following as biased or unequal as to what is given and what is received. Alexander (28) 
claims, 'I'm harvesting a lot, but I'm sowing very little'. Ellen (27) similarly states, ' I'm restrictive with whom I dare to show my music to, but I can take music from a lot of people. I'm like a black hole. I take everything and give very little away'.

Connections made via following in Spotify and Tidal are based on selected friends brought over from Facebook but can also include strangers. Table 2 summarizes the ways in which participants follow strong, weak, and absent ties, and whether the participants' following-patterns primarily are socially or musically motivated.

Table 2: Summary of following patterns from focus groups and diary study.

\begin{tabular}{ll}
\hline Following pattern & Number of participants and motivations for following \\
\hline Strong ties only. & 33 participants, 21 female, 12 male, average age: 26 \\
& Social and music homophily motivate following. \\
Strong, weak, and/or absent ties. & 41 participants, 18 female, 23 male, average age: 29 \\
& Social and/or music homophily motivate following. \\
Weak and or absent ties only. & 26 participants, 10 female, 16 male, average age: 33 \\
& Music homophily motivates following. \\
Do not follow in Spotify or Tidal. & 20 participants, 11 female, 9 male, average age: 28 \\
& Might still receive recommendations via email, \\
& messaging, Facebook, Twitter, genre-specific forums.
\end{tabular}

Uncategorized: 16 participants could not be categorized based on the interviews. 
Following strong ties. Streaming users who follow strong ties in Spotify and Tidal often use the streaming service as a social arena for interacting with friends and maintaining fellowship. In these cases, shared music preferences alone do not trigger following. Rather one seeks to enrich a friendship or gain insight into the strong tie's personal music universe. Strong ties are customarily regarded as safer relations, at least initially.
Albert (30): You can see other people's playlists, and you have a certain idea about the music preferences of people you know, and you can explore a bit based on what you know about these people. Mostly, close friends, really. This might extend over time.

However, social dimensions rarely predict the following of strong ties alone. Shared music taste is the reason why strong ties come to incorporate an aspect of music authoritativeness.

Malin (25): I have perhaps two or three friends with very similar taste in music, and I kind of stalk them on Facebook and ask, 'Is there anything new?' [This happens] both ways, really, (...) we discover music for each other. Particularly my brother, if I see that he's been listening to something new, I know I will like it, and I just throw it into my playlist without checking it out, and then it comes up in my playlist and I'm like, 'What's that? Oh, right, it was that', and it's like a pleasant surprise.

The respect and trust that make some ties strong transfer into musical recognition. Following strong ties is experienced as a safe and convenient way to expand one's music preferences. 
Ingrid (30): I think perhaps the biggest authorities are still those real friends, who are like almost startlingly interested in music.

Mathias (28): No, only my friends, whom I know very well. I somehow think that if they listen to something not typical for 'us', in a way, I respect them as individuals anyway, and I will give them a chance. And it generally turns out to be true, if they listen to something very alternative they consider cool, I usually also find it cool. Don't know if it is because I have a positive attitude toward them.

The trust that is inherent in strong friendships sometimes encourages intense interactions with low thresholds for slipping into competitiveness. Diary participant Nina (27) has a relationship with one of her friends founded on their passionate music interests, yet they sometimes have divergent tastes. She follows him in Spotify to 'keep a little eye on him.' By scanning his playlists and recent tracks, she derives information about what he is doing, which she then uses to her advantage in offline music-related conversations.

Conversely, some informants do not find profit of any sort in following, and prefer talking with friends about music. Still others stress the benefits of acquiring music directly, without bothering to follow anyone.

Camilla (22): I might be somewhat old-fashioned, but I always get recommendations from friends, really. I have one buddy, who for some weird reason knows my musical taste, because every time he recommends something, it's a bull's eye. 
Anders (23): You have to tell me personally, 'You need to listen to this', or else it just disappears in the deluge of things. I don't care if anyone publishes a link to their playlist. Because then it's for everyone and I'm not that interested.

To summarize, following (specific) strong ties is experienced as a trustworthy and valuable way of exploring music. Social and music homophily motivate following, with selected strong ties reflecting both a social bond and 'likes attract' in terms of shared music preferences.

Following weak and absent ties. Following weak ties connects to more exploratory modes of streaming. The comfort of following strong ties gives way to qualified and novel music references that make weak and absent ties into oftentimes amusing and productive sources of new music.

Ellen (27): It's not about personal relationships at all . . it might be rather distant acquaintances. Like, I've just discovered that they [have] much that's interesting.

Julie (40): I don't follow too many that I actually know [because that would be mostly] friends and children's music [laughs]. I follow a few people that I don't know, who I've just come across and who have proven to have great playlists.

Informants follow weak ties selectively without primarily emphasizing the social relationship as reason for the connection. This form of following is instead motivated by perceived music fellowship or recognition, or what we term music homophily. The most knowledgeable listeners in our study regard weak-tie relations as equal peers in terms of matching music interests rather than special friends.

Maria (32): Among my friends, I'm kind of the person who gives recommendations to others, so it's more with people I barely know, or know a 
little, but who I know are kind of at the same place as I am-who like the same music.

Weak ties are regarded as qualified resources for following if they represent expertise or are thought to be up to date. John (35) trusts his group of weak-tie 'music editors' in Spotify. These are peripheral acquaintances who are on the same wavelength when it comes to being nerdy about music. Diary participant Marius (24) follows weak ties that he acknowledges as 'more than averagely interested in music'. He does not necessarily share their tastes but follows them to spark interesting online discussions about music. Thus, whereas musical homophily might initially motivate this kind of following, a sense of belonging and social homophily often come along afterward. People follow weak ties as well because they supply relatively effortless access to music, insights, and recommendations. Following them is a means of benefiting from an extended and knowledgeable network of music-listening peers:

Morten (50): I have three friends who are DJs, who create nice playlists. Yes, 'friends', I have met them, but it's more like Facebook and that world. And there's one, he creates lists before Roskilde [festival]. It's great because then I don't have to.

However, in relation to weak ties, formally following can be characterized by social ambiguity. For diary participant Nina (27), it feels like crossing boundaries to follow certain of her Facebook friends within a streaming service: 'I don't know why, but I'm afraid that if I follow them, they will think: "Why does she add me?" Because I feel I may not know them well enough.' One way out of this quandary is to indirectly follow weak ties by occasionally browsing their streaming accounts. Some participants described indirect following with terms like sneak peeking, lurking, and spying; 
indicating how following also can provide unexpected social insights as well as music recommendations.

Whereas following weak ties can be experienced as socially awkward, following absent ties is liberated from any social expectations. When diary participant Jenny (17) needs music for a specific purpose, she searches for words like 'sleep' and 'exercise'. Spotify accumulates the relevant content for her, and she then helps herself to playlists or selected tracks from strangers, some of whom she might later start to follow. Links to mood- or genre-specific playlists are also often shared on Twitter and other SNSs by people whom streaming users might follow but do not necessarily know. Absent ties thus emerge as valuable sources: '[They are] an excellent way to discover new music. Like if somebody shares a playlist that suits a certain mood, like the "autumn list"' (Jane, 35).

Following absent ties also encompasses connections with music personalities, such as critics, label employees, or musicians. Some users prefer not to choose whom to follow at all, instead relying on algorithms imported from Last.fm or other apps that measure music compatibility. These suggestions, which encompass strong, weak, and absent ties, are thought to be more accurate predictions of potential musical resonance.

To recap, streaming services offer opportunities to discover music through weak and absent ties, which are considered as credible and interesting musical peers. A sense of musical fellowship or music homophily then generally translates into a sense of social belonging. Yet we also see that following weak ties includes experiences of social diffidence to the social relation.

\section{Discussion: Social awareness in sharing and following}

Conclusions about personal experiences are always grounded in the individual and are therefore hard to categorize unambiguously. In addition, it is hard to articulate (and to interpret) music experiences and motivations for sharing and following. Personal 
practices can be inconsistent and sometimes arise without one's conscious awareness as to why or how they came to be. What people say sometimes diverges with what people do and think, and thinking and talking about music can be an act of self-reflexive performance in and of itself. The complex character of this kind of material is reflected in this analysis and underpins the extent and substance of the tables' analytical categories.

With regard to sharing music, our theoretical framework situates people as shaping and staging self-performances according to contexts (Goffman, [1959] 1990), linking with notions of the self as reflexive and partly constituted through social interactions (Blumer, [1969] 1998; Giddens, 1991, Mead and Morris, [1934] 1967). We additionally connect self-performances to taste cultures (DiMaggio, 1987; Tepper and Hargittai, 2009). With regard to social patterns of exploring music, the key theoretical notions relate to homophily (Centola et al., 2007; McPherson et al., 2001) and networked individualism (Rainie and Wellman, 2012). This framework guides the following discussion about what our findings imply for understanding human behaviour with technology.

By integrating social features, music-streaming services inject social transparency into the realm of music listening. Consequently, a tension emerges between expanded everyday uses of music and the service features of sharing and exposing one's multifaceted listening patterns. Put differently, as a consequence of music streaming, the times and places for being social in music listening have grown, as have the range of content exchanged and the list of the social contacts to be included in these music-based interactions. These social features translate into listeners being conscious of whom they relate to and what they listen to. The music experience is thus characterized by social awareness, with regard to being followed and following others. Social awareness in sharing: impression management, control and ontological security Most often social actions are steered by tacit or taken-for-granted qualities, ensuring 
feelings of ontological security (Giddens, 1984; 1991). This sense of ontological security can be threatened when social routines are absent, or in the case of music sharing, when lack of technological control implies the risk of undesired sharing of music taste statements. Non-sharers preserve their ontological security by avoiding the whole possibility. Their social awareness dictates the related verdicts that music is too personal or revealing to be shared, that technology is too transparent, and that sharing as an activity can be inappropriate, unnecessary, or discourteous.

The social awareness of selective sharers prompts them to use social features actively but without the degree of confidence that is required to share everything with everyone. They are deliberate in their use of the technology in order to perform controlled exposures of the self through music. The ways in which the self is contextually performed (Goffman, [1959] 1998) and reflexively maintained through social interaction (Blumer, [1969] 1998; Giddens, 1991, Mead and Morris, [1934] 1967) imply negotiations regarding the shareability of music. Certain music is perceived to have a social bonding-function with close friends, and certain music is perceived to have a value as desired taste statements beyond strong ties. Yet whereas the social advantages of broad cultural preferences are emphasized in the literature in terms of social bridging to weak tie networks (DiMaggio, 1987; Lizardo, 2006; Tepper and Hargittai, 2009), the flatness of the social context in music streaming makes it hard to appropriately deploy heterogeneous taste preferences to context. Technological control is moreover perpetually undermined by software service updates, which often include new features and changes of settings. In our study, the information we could access by monitoring the streaming accounts sometimes surprised the diary participants. After these interviews, we saw playlists being blocked and opened, and settings being changed, all of which is evidence of the fact that social awareness is constantly developing, in line with reflexive self-performances. 
Share-all participants, in contrast, appear to see no need to negotiate music as personal and social. They may consider some of their music as potentially detrimental to their credibility, but not their integrity. As music missionaries, and often taste omnivores, they express little concern with the social need to tailor taste statements to specific context. Instead they emphasize the benefits of sharing; also when sharing implies exposing potentially broad and heterogeneous tastes.

Social awareness in following: homophily

How listeners regard themselves and their peers as musical individuals influences their social conduct online and decisions to follow strong, weak, and absent ties. Following is hence motivated by social awareness to peers in terms of specific expectations. These expectations reflect different notions of homophily.

Social homophily is a useful term for understanding motivations for following strong ties in music streaming. The listener's social awareness is hence based on sociodemographic, behavioural, and intrapersonal characteristics (McPherson et al., 2001). Following that is motivated by social homophily evokes the notion of sociability: playforms of being together, where the association is valued as such, and where being together, rather than the specific content of the communication is of primary interest (Simmel and Wolff, 1964). That said, social homophily is not the only motivation for following strong ties in streaming services. Musical homophily matters too, i.e. these strong tie connections are also characterized by a purpose, moving beyond Simmel's notion of sociability. Similar music interests reflect bonding and trustworthy recommendations among close friends.

Musical homophily promotes following weak and absent ties. Similarity in musical taste is the most common dimension of musical homophily, but one's level of dedication or knowledge is also relevant, even when tastes differ. Absent-tie connections driven by perceived musical homophily are welcomed for the same reason as weak-tie connections; namely, the non-redundant exchange of music information 
originating in an expanded music network. Following weak and absent ties, motivated by musical homophily, can initiate a sense of social homophily in turn, including feelings of belonging to a group or community. This finding confirms Lizardo's claim that networked consumption of widely available cultural goods serves as key to individual connection and integration into social structures (2006, p. 800). In specific, our study demonstrates how the formation of bridges across distant social positions and locally bounded relational clusters (Lizardo, 2006, p. 803) take place in current online music consumption.

The social experience of streaming: Networked individualism and social as a feature Music-streaming services are often used in situations that are considered personal. Also, streaming services archive personally meaningful music compilations. These characteristics place the individual at the centre of the streaming experience, regardless of the social networks that tend to feature so prominently there. As users incorporate their social awareness into their streaming practices, they begin to cultivate 'nuanced understandings of what to make public, which publics to make information available to, and how to intermix technologies of privacy with those of public narrowcasting' (Rainie and Wellman, 2012: 271). Following relations that arise via personal, active choice enhances the social experience more than those that come about via sites or apps that calculate compatibility. Skilled users draw on various aspects of homophily, depending on what they need from diverse types of ties. At the same time, users manifest their social awareness as 'parts of others' networks and they have a heightened sense of obligation to meet the needs of those who consider them social ties' (Rainie and Wellman, 2012: 272).

Relations to peers can be perceived as fundamentally different in terms of sharing versus following. With regard to sharing, in an exploratory listening mode, peers can come across as unsafe in terms of impression management. Yet with regard to following, peers come across as resources of value when exploring music. Positions 
taken as sharers or followers connect with different opportunities and restrictions. People in the networked age are generally free to act on their own, picking and choosing among various segments of their networks (Rainie and Wellman, 2012), but we find different patterns as in how people experience music streaming as social. To some, music listening includes experiences of highly personal dimensions that contrast the social service-features, and the increased time and place of being connected. The experience of social streaming can be risky if it is not controlled adequately, and even intrusive if the time and place for social interaction are not adjusted to one's personal boundaries.

Social networking consumes time (Rainie and Wellman, 2012) and attention, as we have observed here in relation to the impact of social awareness upon the sharing of music. Challenges within this social system must be confronted as they arise, lest some runaway technology disrupt an otherwise carefully cultivated dynamic or persona. Nevertheless, experience with navigating new technology changes over time and with regular use. Confidence in one's music choices and perceived expectations related to personal taste can also evolve, as can social relations, which aligns the personal and social practices of music streaming with the changing character of the technology they use.

\section{Conclusion}

The social features of streaming services enable possibilities for connecting with and being influenced by others. Our findings point to a tension between sharing music and following friends and contacts. Strong, weak, and absent ties appear equally relevant with regard to discovering new music, yet when it comes to sharing music, the trust and confidence that characterize strong ties are crucial. This, however, depends on the ways in which music and sharing relate to identity work. Social awareness is present in sharing and following, in user behaviours that are adapted to listening situations, and in reflections about tie relations. 
Music-streaming services afford several opportunities for users to be connected and to exchange music, yet we continue to question how social the streaming experience actually is. The differences in user patterns and preferences that we have encountered demonstrate heterogeneity among an otherwise relatively homogeneous group of musically inclined and dedicated streaming users. This heterogeneity results from the different exploitations of the opportunities to use music-streaming services, and it is increased by the ways in which streaming use affects social boundaries and impression management. An ongoing, situational negotiation of self and of music as personal or social, and a heightened awareness of others in relation to one's own music listening, are among the social consequences of the use of music-streaming services. We see how fundamental human characteristics sometimes get in the way of the assumed and expected networked benefits of technology. Our findings hence both confirm and nuance Rainie and Wellman's (2012) account of the social operating system of networked individualism, encouraging individuals to share content and obtain information via network structures. Social features integrated into streaming services indeed increase the importance of weak and absent ties for navigating and exploring music. Yet music as personal, connected to taste cultures and impression management, implies consideration of what to share beyond strong ties, to control self-performances in the social network of music streaming.

\section{Limitations and further research}

The main limitation of this study concerns the selection of informants in the focus groups and the diary study, as they were all avid music enthusiasts and users of streaming services in a country with high Internet-penetration and use of smartphones. Our findings do not necessarily reflect the average experience of music-streaming services. This limitation points toward the need for studies with more diverse participants in other countries. 


\section{References}

Baym NK and Ledbetter A (2009) Tunes That Bind? Information, Communication \& Society, 12(3): 408-427. DOI: 10.1080/13691180802635430.

Benkler Y (2006) The Wealth of Networks: How Social Production Transforms Markets and Freedom. New Haven, Conn.: Yale University Press.

Bitner J (ed) (2009) Cassettes from My Ex. New York: St Martin’s Griffin.

Blumer H ([1969] 1998) Symbolic Interactionism: Perspective and Method. Los Angeles: University of California Press.

Brandtzæg PB (2012) Social Networking Sites: Their Users and Social Implications-A Longitudinal Study. Journal of Computer-Mediated Communication 17(4): 467488. DOI: $10.1111 / \mathrm{j} .1083-6101.2012 .01580 . x$.

Centola D, Gonzalez-Avella JC, Eguiluz VM., \& San Miguel M (2007) Homophily, cultural drift, and the co-evolution of cultural groups. Journal of Conflict Resolution, 51(6), 905-929. DOI: 10.1177/0022002707307632.

DeNora T (2000) Music in Everyday Life. Cambridge: Cambridge University Press.

DiMaggio P (1987) Classification in art. American sociological review, 52(4), 440-455.

Ellison N, Steinfield C, and Lampe C (2007) The Benefits of Facebook "Friends": Exploring the Relationship between College Students' Use of Online Social Networks and Social Capital. Journal of Computer-Mediated Communication, 12(4), 1143-1168.

Ezzy D (2002) Qualitative Analysis: Practice and Innovation. London: Routledge.

Giddens A (1984) The Constitution of Society: Outline of the Theory of Structuration. Cambridge: Polity Press.

Giddens A (1991) Modernity and Self-Identity: Self and Society in the Late Modern Age. Cambridge: Polity Press.

Giles DC, Pietrzykowski S, and Clark KE (2007) The Psychological Meaning of Personal Record Collections and the Impact of Changing Technological Forms. 
Journal of Economic Psychology 28(4): 429-443. DOI:

http://dx.doi.org/10.1016/j.joep.2006.08.002.

Goffman E ([1959] 1990) The Presentation of Self in Everyday Life. London: Penguin Press.

Granovetter MS (1973) The Strength of Weak Ties. American Journal of Sociology 78(6): 1360-1380.

Hektner JM, Schmidt JA, and Csikszentmihaly M (2007) Experience Sampling Method: Measuring the Quality of Everyday Life. Thousand Oaks, Calif.: Sage Publications.

Horton D and Wohl RR ([1956] 1986) Mass Communication and Para-Social Interaction: Observation on Intimacy at a Distance. In: Gumpert G and Cathcart $\mathrm{R}$ (eds) Inter/Media: Interpersonal Communication in a Media World. Oxford: Oxford University Press, 3rd ed. pp.185-206.

Ingham, T (2016) YouTube contributed less than 2\% of Norway's streaming income last year. Available at http://www.musicbusinessworldwide.com/youtubecontributed-just-2-of-norways-streaming-income-last-year/ (accessed 3 March 2016).

Jones S (2002) Music That Moves: Popular Music, Distribution and Network Technologies. Cultural Studies 16(2): 213-232. DOI:

10.1080/09502380110107562.

Jones S (2011) Music and the Internet. In: Consalvo M and Ess C (eds) The Handbook of Internet Studies. Oxford: Wiley-Blackwell. pp.440-451.

Kibby M (2009) Collect Yourself. Information, Communication \& Society, 12(3): 428443. DOI: $10.1080 / 13691180802660644$.

Leong TW and Wright PC (2013) Revisiting Social Practices Surrounding Music. In: SIGCHI Conference on Human Factors in Computing Systems (CHI '13), Paris, France: ACM Press. pp.951-960. 
Liu KT and Reimer RA (2008) Social Playlist: Enabling Touch Points and Enriching Ongoing Relationships through Collaborative Mobile Music Listening. In: Proc. Mobile HCI '08, pp.403-406. New York, USA: ACM Press.

Lizardo O (2006) How cultural tastes shape personal networks. American Sociological Review, 71(5), 778-807. DOI: 10.1177/000312240607100504.

Marwick AE and boyd, d (2011) I tweet honestly, I tweet passionately: Twitter users, contex collapse, and the imagined audience. New Media \& Society 13(1): 114133.

McKenna KYA, Green AS, and Gleason MEJ (2002) Relationship Formation on the Internet: What's the Big Attraction? Journal of Social Issues 58(1): 9-31.

McPherson M, Smith-Lovin L, and Cook JM (2001) Birds of a Feather: Homophily in Social Networks. Annual Review of Sociology 27(1): 415-444. DOI:

10.1146/annurev.soc.27.1.415.

Mead GH and Morris CW ([1934] 1967) Mind, Self, and Society: From the Standpoint of a Social Behaviorist. Chicago: University of Chicago Press.

Mesnage, CS, Rafiq, A., Dixon, S. and Brixtel, RP. (2011) Music discovery with social networks. In Workshop on Music Recommendation and Discovery (pp. 1-6).

Rainie H and Wellman B (2012) Networked: The New Social Operating System. Cambridge, Mass.: MIT Press.

Simmel G and Wolff KH (1964) The Sociology of Georg Simmel. New York: Free Press.

Turino T (1999) Signs of Imagination, Identity, and Experience: A Peircian Semiotic Theory for Music. Ethnomusicology 43(2): 221-255.

Van Dijck J (2007) Mediated Memories in the Digital Age. Stanford, Calif.: Stanford University Press. 
Voida A, Grinter RE, Ducheneaut N, Edwards WK, and Newman MW (2005) Listening in: Practices Surrounding iTunes Music Sharing. In: Proc. CHI2005: 191-200. DOI: $10.1145 / 1054972.1054999$.

Yin, R. K. (2009). Case Study Research: Design and Methods (4th ed.). Los Angeles, Calif.: Sage Publications. 